\title{
A Metal Object Detection System with Multilayer Detection Coil Layouts for Electric Vehicle Wireless Charging
}

\author{
Bo Cheng ${ }^{1,2}$, Jianghua Lu ${ }^{1,3}$, Yiming Zhang ${ }^{1}$, Guang Pan ${ }^{2}$, Rakan Chabaan ${ }^{4}$ and \\ Chunting Chris Mi ${ }^{1, *(1)}$ \\ 1 Department of Electrical and Computer Engineering, San Diego State University, San Diego, CA 92182, USA; \\ chengbov@nwpu.edu.cn (B.C.); tri_lih@whut.edu.cn (J.L.); zhangym07@gmail.com (Y.Z.) \\ 2 School of Marine Science and Technology, Northwestern Polytechnical University, Xi'an 710072, China; \\ panguang@nwpu.edu.cn \\ 3 School of Electrical Engineering, Wuhan University of Technology, Wuhan 430070, China \\ 4 Hyundai-Kia America Technical Center, Inc., Superior Township, MI 48198, USA; rchabaan@hatci.com \\ * Correspondence: mi@ieee.org
}

Received: 15 May 2020; Accepted: 5 June 2020; Published: 9 June 2020

\begin{abstract}
Non-radiative inductive power transfer is one of the most studied and commercially applied wireless charging technologies, where the magnetic field is employed as the medium for power transfer. In the wireless charging of electric vehicles, the strong magnetic field will heat up any metal items falling in the charging area due to eddy current induced in the metal objects, causing hazards like fire. Metal object detection (MOD) is necessary for the market penetration of inductive power transfer technology. This paper aims to improve the performance of systems that detect metal objects based on inductance variations. Two novel multi-layer detection coil layouts are proposed, which can not only cover the entire charging area without blind spots but can also be decoupled from the transmitter and receiver to minimize the influence of the magnetic field that is used for power transfer. Two mixed resonant circuits are proposed and proven to have better performance than parallel and series resonance. The impacts of the detection coil layer, trace width, and turn-number are investigated. The test results indicate that the MOD system can detect one-cent coins at various positions of the detection coil printed circuit board, and can also detect various inductance variations without blind spots in the processing circuit.
\end{abstract}

Keywords: multi-layer detection coil; inductance variation; metal object detection; wireless charging; inductive power transfer

\section{Introduction}

Wireless power transfer (WPT) is the emerging technology that can potentially provide convenient charging without cables [1-3]. WPT via near-field magnetic induction, or non-radiative inductive power transfer, is one of the most studied and commercially applied technologies among all WPT technologies [4-7]. One of the typical applications of inductive power transfer is the wireless charging of electric vehicles (EVs) [8-10], which can achieve charging automation and convenience, especially for future autonomous vehicles. However, the strong magnetic field that the WPT system relies on for power transfer can induce strong eddy currents in the metal objects (MOs) falling in the charging area, leading to hazards like fire. On the other hand, the charging area of a wireless EV charging system is normally large, allowing living objects (LOs), such as cats, dogs, and toddlers, to enter the area, causing danger to them. MOs and LOs are foreign objects (FOs) and a wireless EV charging system should be able to detect them. 
Foreign object detection (FOD) technology is crucial to the safe operation of a wireless EV charging system. FOD can be divided into metal object detection (MOD) and living object detection (LOD). There are two major methods of FOD in a wireless EV charging system. The first method is called the wave-based detection method, in which wave-based devices, such as radar [11-13], ultrasonic transducer [14], video camera [14,15], and thermal camera [14,16], are employed to detect FOs. The precision of this method is dependent on the device and signal processing, which is high in cost.

The other method is called the field-based detection method, in which the variation of resistance, inductance, or capacitance is utilized to indicate the existence of an FO. The variation of these electric quantities can also lead to the variation of some other electric quantities, such as quality factor [17], induced voltage [18,19], mutual inductance [20], and resonant frequency [21]. These parameters can also be used for object detection. The field-based detection method can be further divided into inductive sensing and capacitive sensing. The inductive sensing is based on inductance variation and is normally used for MOD. A detection coil array is added on the surface of the transmitter to detect the existence of an MO. The capacitive sensing is based on capacitance variation and is normally used for LOD [22]. The categories of FOD technology are shown in Figure 1.

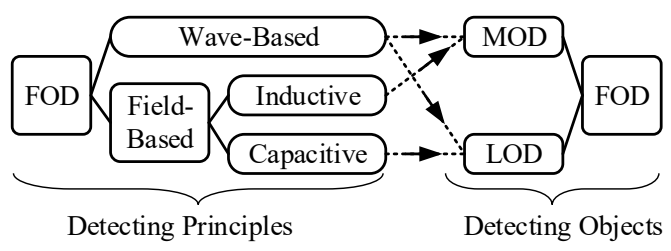

Figure 1. Category of foreign object detection (FOD), metal object detection (MOD) and living object detection (LOD) technology.

In the field-based detection method, via inductive sensing, there are two major methods. The first one is the passive detection method, in which the magnetic field of power transfer is utilized to generate induced voltages on the detection coil, which is affected by MOs. In Reference [18], a coil set consisting of two unipolar coils is used as the detection coil. Without an MO, the induced voltages on these two coils would be the same. The existence of an MO would lead to the imbalance of the induced voltages, which can be used to detect the MO. There is no external source and no impact from the field of power transfer. However, there are many blind spots and it can only perform on-line detection and the power transfer must be on.

The other method is active detection method, where an external source is applied to one detection coil and the parameter variation on this detection coil [23] or another [24] is employed to indicate the existence of an MO. The advantages of this method include fewer blind zones than the passive detection method and the ability to conduct off-line detection. However, an external source is required, and the magnetic field of the power transfer will impact the precision of MO detection. The impact can be alleviated by decoupling the detection coils from the transmitter coil (TX) and filtering the signals from power transfer.

The active detection method based on inductance variations of detection coils is adopted. Compared with Reference [23], novel coil array layouts are proposed in this paper to not only cover the whole area, including the boundary area of each detection coil, but the detection coils are also decoupled from the TX. The mixed resonant circuits are studied to amplify the inductance variation for better detection performance. The impacts of the coil layer, trace width, and turn number are investigated. Experiments are conducted to validate the proposed detection method.

\section{Detection Coil Array}

Because the TX is a unipolar coil, to minimize the impact of the magnetic field from power transfer, the detection coil should be decoupled from the TX. The detection coil can be designed as a bipolar 
coil, which consists of two unipolar coils with opposite polarities connected in series. The idea of decoupling is to let these two unipolar coils have opposite amounts of magnetic flux so that the induced voltages on these two unipolar coils by the field of the main coils can cancel each other out.

In order to minimize the area of blind spots, the two unipolar coils forming a bipolar coil should not be adjoining. Otherwise, the area around the common line or point would be the blind spot, because around this area, the MO would have approximately the same impact on these two unipolar coils and the parameter variation would be negligible. In addition, since the parameter variation in the boundary areas is weak, the boundary area of a detection coil should be overlapped by the center area of another detection coil. Thus, an MO can be guaranteed to be around the center area of one of the detection coils.

\subsection{Three-Layer Hexagonal Layout Detection Coils}

In consideration of the above principles, a hexagonal cell layout for the detection coils is proposed for the MOD system, as shown in Figure 2a. The detection coils are placed in the hexagonal cell arrangement. The centers of the detection coils are fixed at the centers of the hexagons. The detection coil length and shape can be varied. The effective detecting area of each coil should at least cover the hexagon area. Thus, the whole area can be detectable. In this paper, the actual coil shape is a square coil, shown in Figure 2b. Coils with the same color are on the same layer. The two unipolar coils marked with the same number are connected in series with opposite polarities to form a bipolar coil to achieve decoupling from the TX. Thus, bipolar coils $1 \sim 6$ are formed. Bipolar coils 1 and 2 are on the top layer, 3 and 4 on the middle, and 5 and 6 on the bottom. A fourth layer is added for routing and connection. For better understanding, the routing of bipolar coil 1 is shown in Figure $2 b$, where the dotted line indicates the routing on the fourth layer. Therefore, the detection coil board is a four-layer printed circuit board (PCB).

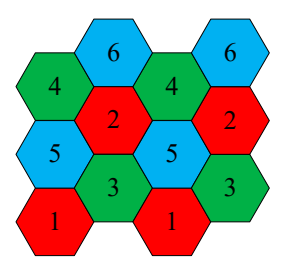

(a)

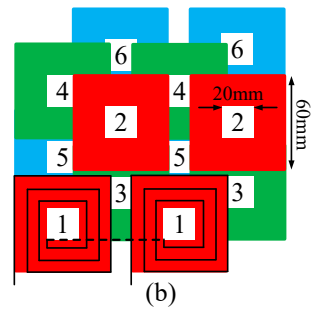

(b)

Figure 2. Detection coil layout. (a) Positions and arrangements of each coil, (b) actual coil shape and routing.

The outer length of the unipolar coil is set to $60 \mathrm{~mm}$ and the inner length is $20 \mathrm{~mm}$. A quarter of the detection coils are built in the simulation software Ansys Maxwell, shown in Figure 3a, since it is symmetrical in the two orthogonal directions. Figure $3 \mathrm{~b}$ is half of the magnetic field distribution between the transmitter and receiver. The self-inductance of the detection coil simulated by Maxwell is $14.2 \mu \mathrm{H}$. The maximum coupling coefficient between the detection coil and the TX simulated by Maxwell is $1.6 \%$. Therefore, the decoupling is achieved. 


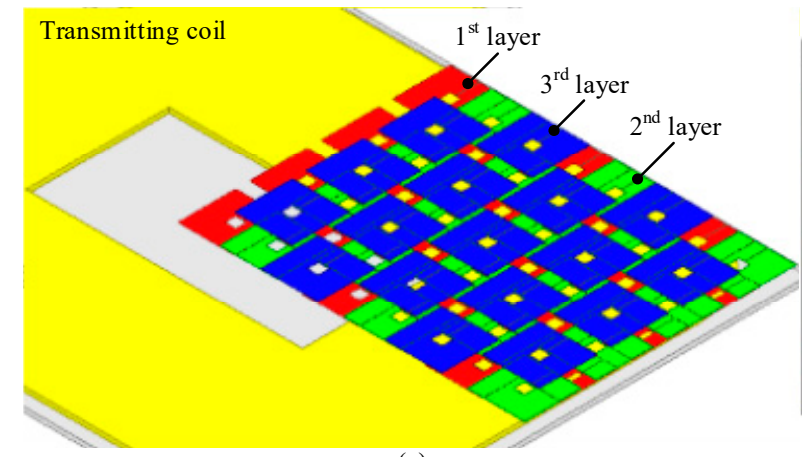

(a)

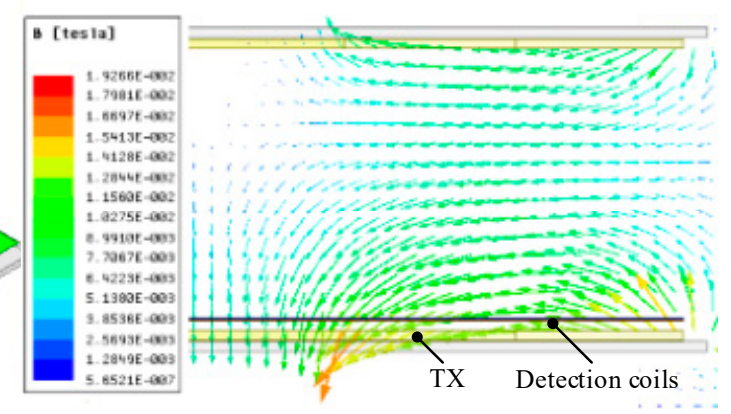

(b)

Figure 3. (a) Model of a quarter of the three-layer hexagonal layout detection coils in Maxwell. (b) Half of the magnetic field distribution between the transmitter and receiver.

\subsection{Four-Layer Rectangular Layout Detection Coils}

Rectangle layout for the detection coils is also proposed for the MOD system. A half of the proposed four-layer rectangle layout detection coil array in Maxwell is shown in Figure 4a. The structure and parameters of the first layer detection coils are shown in Figure $4 \mathrm{~b}$. Two unipolar detection coils of the first layer $A_{11}$ and $A_{12}$ are connected in series with opposite polarity to form the bipolar detection coil $A_{1}$, as shown in Figure $4 b$. Similarly, the unipolar detection coils $A_{n 1}$ and $A_{n 2}$ are connected to form the bipolar detection coil $A_{n}$. The self-inductance of the detection coil simulated by Maxwell is $22.2 \mu \mathrm{H}$. In the proposed four-layer overlapping detection coil array, the edge region of a detection coil can be covered by the region near the center of detection coils in other layers, so the edge regions of the detection coils are the overlapping area, as shown in Figure 4a. In this way, the blind spots at the edge of the detection coils can also be eliminated. The four-layer PCB can be used to implement the four-layer detection coil array, and the gaps between the detection coils of one layer are used for wiring and routing of the detection coils in other layers.

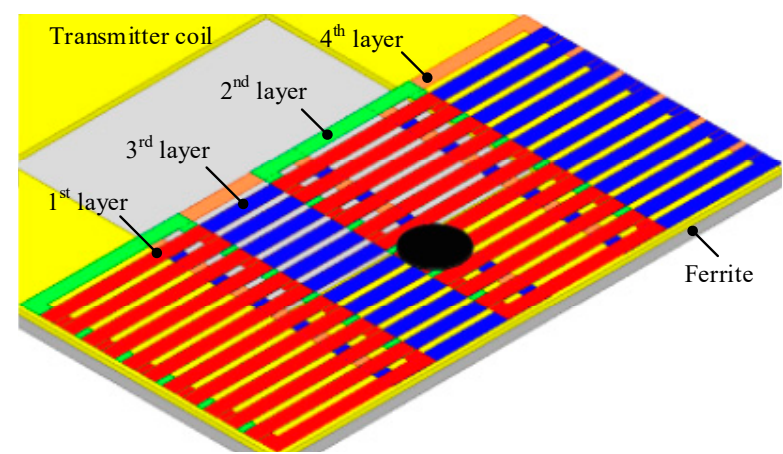

(a)

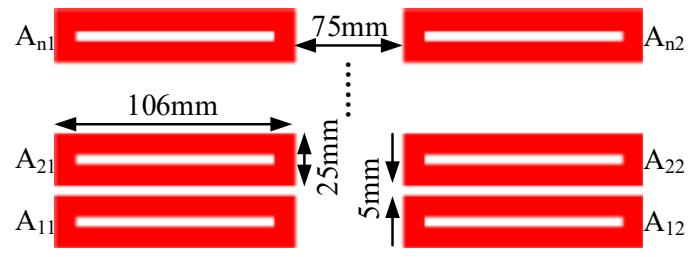

(b)

Figure 4. (a) Model of a half of the four-layer rectangular layout detection coils in Maxwell. (b) The Structural parameters of the first layer detection coils.

\section{Principle of Detection}

\subsection{Inductance Variation}

When an $\mathrm{MO}$ is placed above the detection coil, as shown in Figure 5a, the parameters of the detection coil change. The equivalent circuit is given in Figure $5 \mathrm{~b}$. The $\mathrm{MO}$ can be regarded as an inductance $L_{\mathrm{MO}}$ in series with its own equivalent resistance $R_{\mathrm{MO}}$, coupled with the detection coil. $L_{\text {det }}$ and $R_{\text {det }}$ are the inductance and resistance of the detection coil, respectively. $M$ is the mutual 
inductance between the detection coil and the MO, which can be calculated explicitly [25]. $L_{\text {detMO }}$ and $R_{\text {detMO }}$ are the equivalent inductance and resistance of the detection coil with the MO, respectively.

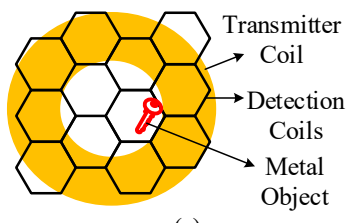

(a)

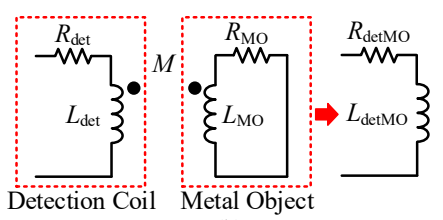

(b)

Figure 5. Detection coil with an MO. (a) Schematic, (b) equivalent circuit.

The input impedance of the detection coil with the MO is:

$$
Z_{\mathrm{in}}=R_{\mathrm{det}}+\mathrm{j} \omega L_{\mathrm{det}}+\frac{(\omega M)^{2}}{R_{\mathrm{MO}}+\mathrm{j} \omega L_{\mathrm{MO}}}=\left(R_{\mathrm{det}}+\frac{(\omega M)^{2} R_{\mathrm{MO}}}{R_{\mathrm{MO}}^{2}+\left(\omega L_{\mathrm{MO}}\right)^{2}}\right)+\mathrm{j} \omega\left(L_{\mathrm{det}}-\frac{(\omega M)^{2} L_{\mathrm{MO}}}{R_{\mathrm{MO}}^{2}+\left(\omega L_{\mathrm{MO}}\right)^{2}}\right)
$$

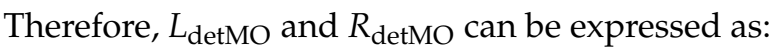

$$
\begin{gathered}
L_{\mathrm{det} M O}=L_{\mathrm{det}}-\frac{(\omega M)^{2} L_{\mathrm{MO}}}{R_{\mathrm{MO}}^{2}+\left(\omega L_{\mathrm{MO}}\right)^{2}}=L_{\mathrm{det}}(1-\alpha) \\
R_{\mathrm{detMO}}=R_{\mathrm{det}}+\frac{(\omega M)^{2} R_{\mathrm{MO}}}{R_{\mathrm{MO}}^{2}+\left(\omega L_{\mathrm{MO}}\right)^{2}}=R_{\mathrm{det}}(1+\beta)
\end{gathered}
$$

where $\alpha$ and $\beta$ are the variation ratio of the inductance and the resistance, expressed as:

$$
\begin{gathered}
\alpha=\frac{(\omega M)^{2} L_{\mathrm{MO}}}{\left[R_{\mathrm{MO}}^{2}+\left(\omega L_{\mathrm{MO}}\right)^{2}\right] L_{\mathrm{det}}} \\
\beta=\frac{(\omega M)^{2} R_{\mathrm{MO}}}{\left[R_{\mathrm{MO}}^{2}+\left(\omega L_{\mathrm{MO}}\right)^{2}\right] R_{\mathrm{det}}}
\end{gathered}
$$

As can been see from Equations (2) and (3), the equivalent inductance of the detection coil decreases by $\alpha$ and the equivalent resistance increases by $\beta$ with the presence of an MO. A larger $M$ results in a larger inductance variation. That means that when the $\mathrm{MO}$ is close to and located at the center of the detection coil, $M$ is large and so is the inductance variation.

\subsection{Mixed Resonances}

\subsubsection{SP Mixed Resonances}

Even though the inductance and resistance changes due to the existence of an $\mathrm{MO}$, the variation itself is quite small and the measurement of these variations can be difficult. Therefore, a resonant circuit can be applied to the detection coil to amplify the inductance variation. Basically, there are two resonant topologies: series resonance and parallel resonance. They were analyzed and compared in Reference [23] and parallel resonance was selected due to its lower sensitivity to noise and lower power consumption when there is no MO. On the basis of parallel resonance, the capacitor in series with a detection coil was added to form a mixed resonance, where the series connected capacitor $C_{S}$ and the parallel connected capacitor $C_{P}$ resonate with the detection coil together, as shown in Figure 6. This mixed resonance is called the SP mixed resonance in this paper. However, Reference [23] has not revealed how to select $C_{P}$ and $C_{S}$ so as to maximize the variation. 


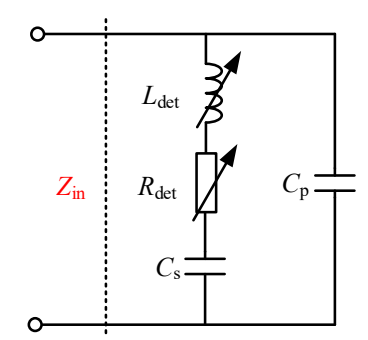

Figure 6. SP mixed resonance to amplify inductance variation.

Define

$$
\lambda=\frac{C_{\mathrm{S}}}{C_{\mathrm{P}}+C_{\mathrm{S}}}
$$

The resonant angular frequency of the SP mixed resonant circuit is:

$$
\omega_{0}=\frac{1}{\sqrt{L_{\operatorname{det}} C_{P} \| C_{S}}}=\frac{1}{\sqrt{L_{\operatorname{det}} \frac{C_{P} C_{S}}{C_{P}+C_{S}}}}=\frac{1}{\sqrt{L_{\operatorname{det}} \lambda C_{P}}}
$$

Without MOs, the input impedance $Z_{\text {in }}$ at $\omega_{0}$ is:

$$
Z_{\text {in }}\left(\omega_{0}\right)=\frac{\frac{1}{j \omega_{0} C_{P}}\left(\frac{1}{j \omega_{0} C_{S}}+j \omega_{0} L_{\text {det }}+R_{\text {det }}\right)}{\frac{1}{j \omega_{0} C_{P}}+\left(\frac{1}{j \omega_{0} C_{S}}+j \omega_{0} L_{\text {det }}+R_{\text {det }}\right)}=\lambda \omega_{0} L_{\text {det }}\left(\lambda Q_{\text {det }}-j\right)
$$

where $Q_{\text {det }}$ is the quality factor of the detection coil, defined as:

$$
Q_{\mathrm{det}}=\frac{\omega_{0} L_{\mathrm{det}}}{R_{\mathrm{det}}}
$$

With the presence of an $\mathrm{MO}$, assume $L_{\mathrm{detMO}}=(1-\alpha) L_{\mathrm{det}}$ and ignore the variation of the equivalent resistance for simplicity. The input impedance can be calculated as:

$$
Z_{\mathrm{inMO}}\left(\omega_{0}\right)=\lambda \omega_{0} L_{\mathrm{det}}\left(\lambda \frac{Q_{\mathrm{det}}+\alpha Q_{\mathrm{det}}^{2}}{1+\left(\alpha Q_{\mathrm{det}}\right)^{2}}-\mathrm{j}\right) .
$$

For proportional amplifier circuits or voltage division circuits, it is the impedance amplitude that affects the output. Therefore, the impedance amplitude variation is studied. The ratio of the input impedance amplitude difference $\delta$ is defined as:

$$
\delta=\frac{\left|Z_{\text {inMOO }}\right|-\left|Z_{\text {in }}\right|}{\left|Z_{\text {in }}\right|}
$$

where $\delta$ can be the indicator for the system performance. The larger the absolute value of $\delta$, the better the system performance.

When the angular frequency is $\omega_{0}$, the amplitude of the input impedance without and with an MO can be expressed as:

$$
\begin{gathered}
\left|Z_{\text {in }}\left(\omega_{0}\right)\right|=\lambda \omega_{0} L_{\text {det }} \sqrt{1+\left(\lambda Q_{\text {det }}\right)^{2}} \\
\left|Z_{\text {inMO }}\left(\omega_{0}\right)\right|=\lambda \omega_{0} L_{\text {det }} \sqrt{1+\left(\lambda Q_{\text {det }}\right)^{2} \frac{1-2 \frac{\alpha}{\lambda}}{1+\left(\alpha Q_{\text {det }}\right)^{2}}}
\end{gathered}
$$

Comparing Equation (12) with Equation (13), the existence of $\alpha$ in the second term under the square root of Equation (13) reduces the amplitude due to the increase of the denominator and decrease 
of the numerator. The larger the $\alpha$, the larger the amplitude variation. In addition, the smaller the $\lambda$, the larger the amplitude variation. The larger the $Q_{\text {det }}$, the larger the amplitude variation.

In the study of the SP mixed resonance, the detection coils are three-layer hexagonal layout detection coils. According to Maxwell simulation results, $L_{\text {det }}$ is set to $14.2 \mu \mathrm{H}, R_{\text {det }}$ is set to $3.6 \Omega$, and the resonant frequency is set to $1 \mathrm{MHz}$. The relationship between $\delta$ and $\lambda$ under different values of $\alpha$ and $\beta$ is shown in Figure 7. With the decrease of $\lambda$, the absolute value of $\delta$ is increasing, which validates that the smaller the $\lambda$, the larger the variation, and the better the system performance. However, if $\lambda$ is too small, the system input impedance would not be high, and the SP mixed resonant circuit would not be as insensitive to the noise as the parallel resonant circuit. Therefore, $\lambda$ should be properly selected according to the designed system.

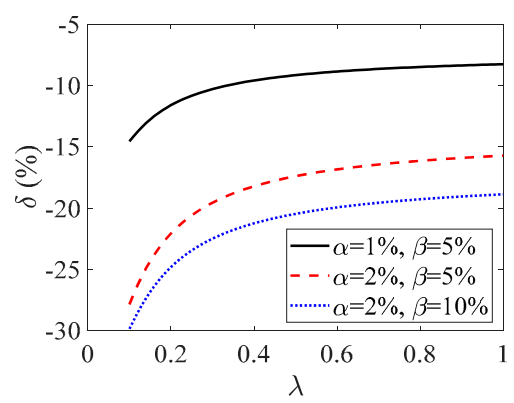

Figure 7. $\delta$ changes with $\lambda$ under different values of $\alpha$ and $\beta$.

Based on the calculation setup above, $\lambda$ is set to 0.2 , and how $\delta$ changes with the operating frequency $f$ is shown in Figure 8. The maximum variation, which is the valley value of $\delta$, occurs at the frequency point slightly smaller than the resonant frequency.

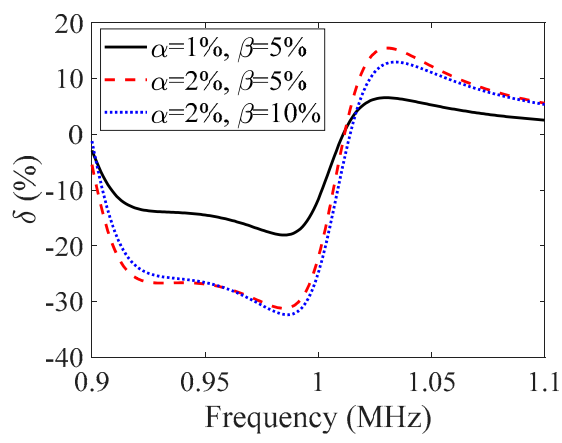

Figure 8. $\delta$ changes with $f$ under different values of $\alpha$ and $\beta$, for the SP mixed resonance, when $\lambda=0.2$.

From both Figures 7 and 8, it can be seen that the amplitude variation is more sensitive to the inductance variation than to the resistance variation.

\subsubsection{PS Mixed Resonances}

PS mixed resonance is defined when a capacitor is added in series with the parallel resonance of the detection coil, as shown in Figure 9, it is called PS mixed resonance in this paper. In the case of PS mixed resonance, neglecting the detection coil resistance, the resonant angular frequency is:

$$
\omega_{0}=\frac{1}{\sqrt{L_{\operatorname{det}}\left(C_{S}+C_{P}\right)}}
$$




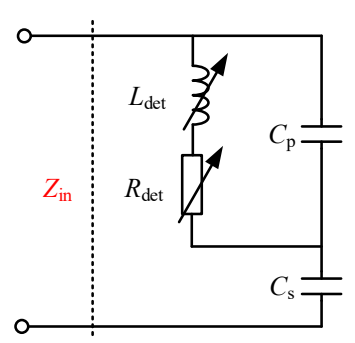

Figure 9. PS mixed resonance to amplify inductance variation.

The input impedance of the resonant circuit without MOs on the detection coils can be expressed as:

$$
Z_{\text {in }}(\omega)=\frac{\frac{1}{j \omega C_{\mathrm{P}}}\left(j \omega L_{\mathrm{det}}+R_{\mathrm{det}}\right)}{\frac{1}{j \omega C_{\mathrm{P}}}+\left(\mathrm{j} \omega L_{\mathrm{det}}+R_{\mathrm{det}}\right)}+\frac{1}{\mathrm{j} \omega C_{\mathrm{S}}}
$$

In the study of the PS mixed resonance, the detection coils are four-layer rectangular layout detection coils. According to Maxwell simulation results, $L_{\operatorname{det}}$ is set to $22.2 \mu \mathrm{H}, R_{\operatorname{det}}$ is set to $4.9 \Omega$, the resonance frequency is set to $1 \mathrm{MHz}, C_{\mathrm{S}}$ is set to $0.2 \mathrm{nF}$, and $C_{\mathrm{P}}$ is $0.94 \mathrm{nF}$. When $\alpha$ is $4.7 \%$, the input impedance of the resonant circuit and $\delta$ change with the operating frequency $f$ is shown in Figure 10. The maximum variation, which is the valley value of $\delta$, occurs at the frequency point slightly larger than the resonant frequency. The proposed PS mixed resonant circuit not only has a high impedance, but also has a large impedance variation ratio.

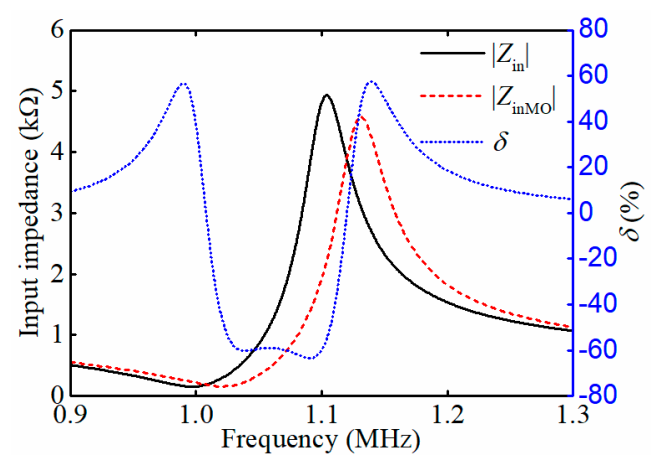

Figure 10. The input impedance of the resonant circuit and $\delta$ change with the operating frequency $f$.

\subsection{System Topology}

The overall circuit of the MOD system is shown in Figure 11. The SP and PS mixed resonant circuits can both be adopted to amplify the inductance variation. The PS mixed resonant circuit is shown in Figure 11. $C_{\mathrm{P}}$ is the parallel capacitance, $C_{\mathrm{S}}$ the series capacitances, $B S_{11}$ and $B S_{12}$ constitute a bidirectional switch, and so do $B S_{21}$ and $B S_{22}, \cdots, B S_{\mathrm{n} 1}$ and $B S_{\mathrm{n} 2}$. The bidirectional switches are used to select one branch of detection coil at a time. $U_{S}$ is a high-frequency alternating current (AC) source, $R_{\mathrm{S}}$ is the divider resistance, and $U_{\text {det }}$ is the voltage on the resonant circuit at the operating frequency, as:

$$
U_{\text {det }}=\frac{\left|Z_{\text {in }}\right|}{\left|R_{\mathrm{S}}+Z_{\text {in }}\right|} U_{S}
$$

In this way, the inductance variation of the detection coil $\alpha$ is converted into the voltage variation. Despite the use of bipolar detection coils, it is difficult to completely cancel out the induced voltage in the detection coils, so a third order high-pass filter is used to filter out the noise near the power transfer frequency. A diode is utilized to convert the AC output voltage into the direct current (DC) 
output voltage, $U_{\text {out }}$, so that a low-cost voltage sensor can be used to measure the DC output voltage. The voltage gain of the overall system is defined as:

$$
G_{U}=\frac{U_{\text {out }}}{U_{\mathrm{S}}}
$$

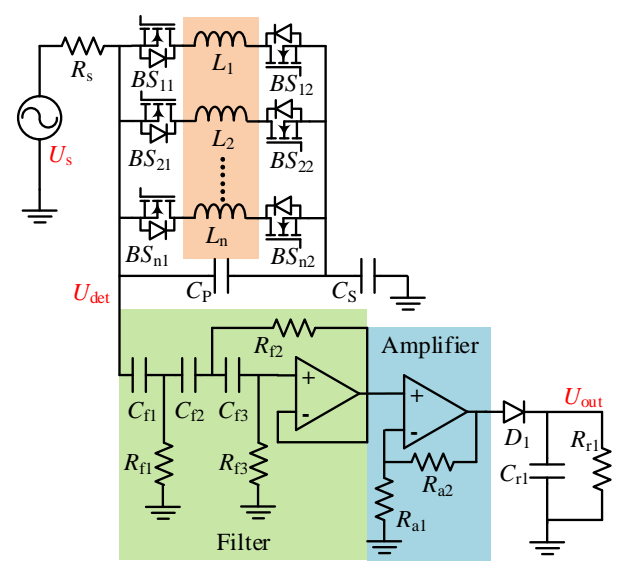

Figure 11. System topology.

This voltage gain is the indicator of the existence of an MO. The voltage gain variation is used to measure the degree of variation for cases without and with an $\mathrm{MO}$, and is defined as:

$$
\gamma=\frac{G_{U \mathrm{w} /}-G_{U \mathrm{wo}}}{G_{U \mathrm{wo}}}=\frac{U_{\mathrm{det} \mathrm{MO}}-U_{\mathrm{det}}}{U_{\operatorname{det}}}
$$

where, $G_{U \text { wo }}$ is the voltage gain without an $\mathrm{MO}$ and $G_{U \mathrm{w} /}$ is the voltage gain with an $\mathrm{MO}, U_{\mathrm{det}} \mathrm{MO}$ is the voltage on the resonant circuit with an $\mathrm{MO}$.

Figure 12 shows $\gamma$ versus $\alpha$ at three different operating frequencies of the PS mixed resonant circuit. Here, a negative $\alpha$ indicates the reduction in the detection coil inductance, and a positive $\alpha$ indicates the increase in the detection coil inductance. As can be seen from Figure 10, the maximum impedance variation occurs in the frequency range slightly larger than the resonance frequency, and three operating frequencies are selected from this frequency range. When the operating frequency is $1.02 \mathrm{or} 1.12 \mathrm{MHz}$, there is a blind spot where the output voltage does not change. When the operating frequency is 1.07 $\mathrm{MHz}$, there is no blind spot for detecting various inductance variation. The proper operating frequency should be lower than the frequency where the impedance of the resonant circuit is maximal, as shown in Figure 10. But, if the operating frequency is much lower than the frequency, it will still cause blind spots. Therefore, the proper operating frequency should be slightly lower than the frequency where the input impedance of the resonant circuit is maximal. In this way, the reasonable operating frequency can be selected to eliminate the blind spot in the processing circuit.

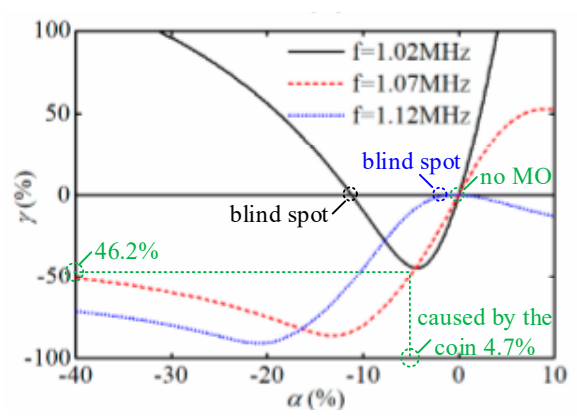

Figure 12. $\gamma$ versus $\alpha$ at three different operating frequencies of the PS mixed resonant circuit. 


\section{System Implementation and Measurement}

\subsection{Impacts of Detection Coil Parameters}

The research on the impacts of the detection coil parameters in this subsection is based on the MOD system of the three-layer hexagonal layout detection coil array and the SP mixed resonance circuit.

Based on Figure 2, a PCB detection coil is designed and fabricated with its layout shown in Figure 13. The two unipolar coils encircled by a rectangle form a bipolar coil. The coil outer length is $60 \mathrm{~mm}$ and the inner length is $20 \mathrm{~mm}$. The other coil parameters of these six bipolar coils are given in Table 1 . The inductance is measured when the detection coil board is placed on the TX with ferrite. Coil 5 has the largest inductance value among Coils 1, 2, 3, and 5 because it is on the bottom layer and is closest to the ferrite.

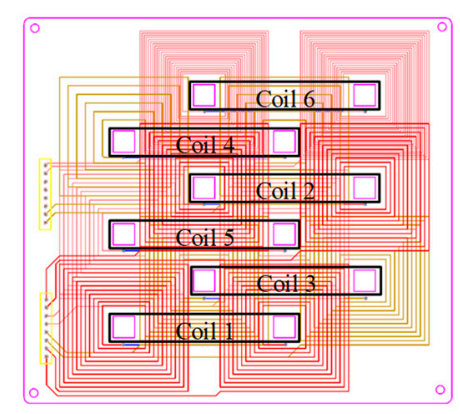

Figure 13. Printed circuit board (PCB) layout of the three-layer hexagonal layout detection coils.

Table 1. Detection coil parameters.

\begin{tabular}{ccccc}
\hline Coils & Layer & $\begin{array}{c}\text { Trace } \\
\text { Width }(\mathbf{m m})\end{array}$ & $\begin{array}{c}\text { Turn } \\
\text { Number }\end{array}$ & $\begin{array}{c}\text { Measured } \\
\text { Inductance }(\boldsymbol{\mu H} \mathbf{H})\end{array}$ \\
\hline Coil 1 & 1 & 0.5 & 11 & 14.54 \\
Coil 2 & 1 & 0.3 & 11 & 14.69 \\
Coil 3 & 2 & 0.5 & 11 & 14.56 \\
Coil 4 & 2 & 0.5 & 6 & 5.01 \\
Coil 5 & 3 & 0.5 & 11 & 15.13 \\
Coil 6 & 3 & 0.5 & 21 & 53.22 \\
\hline
\end{tabular}

A United States (US) one-cent coin is selected as the MO, shown in Figure 14a. The positions of the MO are defined in Figure 14b. The best and worst positions of the bipolar coil are at the center and corner (boundary position) of one of the unipolar coils, respectively. When the MO is placed further away from the boundary position, another bipolar coil can be selected for the MO to be considered to have a better position.

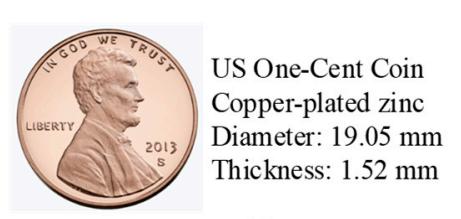

(a)

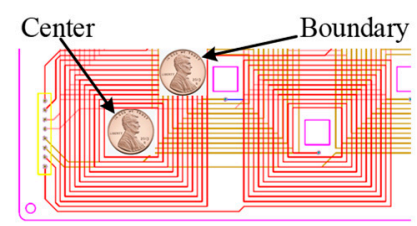

(b)

Figure 14. MO. (a) Photograph, (b) positions.

To investigate the impact of $\lambda$, different combinations of $C_{P}$ and $C_{\mathrm{S}}$ are selected. Take Coil 1 as an example. As can be seen from Figure 7 , the smaller the $\lambda$, the larger the impedance variation. Three $\lambda$ are used to verify the impact of $\lambda$ on the system performance, and $\lambda$ depends on the combinations of $C_{P}$ and $C_{S}$. When $C_{S}$ is eliminated and $C_{P}=1.60 \mathrm{nF}, \lambda=1$; when $C_{S}=8.48 \mathrm{nF}$ and $C_{P}=1.97 \mathrm{nF}$, 
$\lambda=0.81$; when $C_{\mathrm{S}}=1.97 \mathrm{nF}$ and $C_{\mathrm{P}}=8.48 \mathrm{nF}, \lambda=0.19$. Figure 15 shows the voltage gain variation $\gamma$ changing with the operating frequency under different values of $\lambda$ when the coin is placed at the center position. A smaller $\lambda$ leads to a larger $\gamma$, which validates the analysis in Section 3.2.1. The following experiments are all conducted when $\gamma$ is around 0.2 .

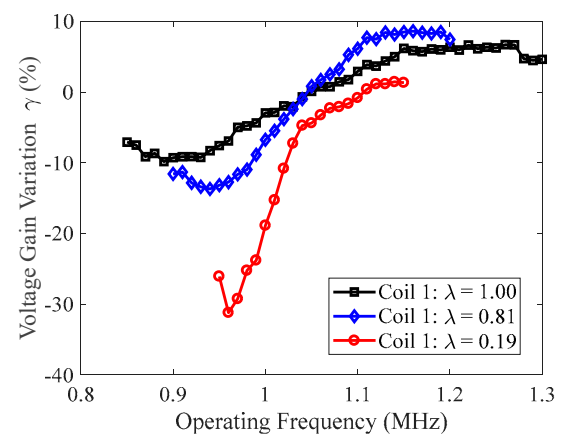

Figure 15. Measurements of $\gamma$ varying with $f$ under different values of $\lambda$ for Coil 1 .

To investigate the impact of different layers of the PCB, Coils 1,3 , and 5 are compared. $C_{S}=1.97 \mathrm{nF}$ and $C_{\mathrm{P}}=8.48 \mathrm{nF}$. The measurements of these three coils when the coin is placed at the center position are shown in Figure 16. Coil 1 at the top layer has the largest variation because it is closest to the coin vertically. Coil 3 at the middle layer has the second largest variation and Coil 5 at the bottom layer has the smallest variation.

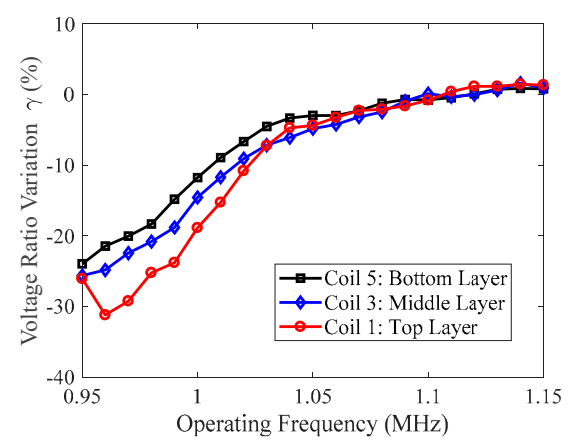

Figure 16. Measurements of $\gamma$ varying with $f$ for Coils 1,3 , and 5 .

To investigate the impact of trace width, Coil 1 and Coil 2 are compared. $C_{\mathrm{S}}=1.97 \mathrm{nF}$ and $C_{\mathrm{P}}=8.48 \mathrm{nF}$. The measurements of the two coils when the coin is placed at the center position are shown in Figure 17. Coil 1 has a larger voltage gain variation due to its larger quality factor caused by the larger trace width.

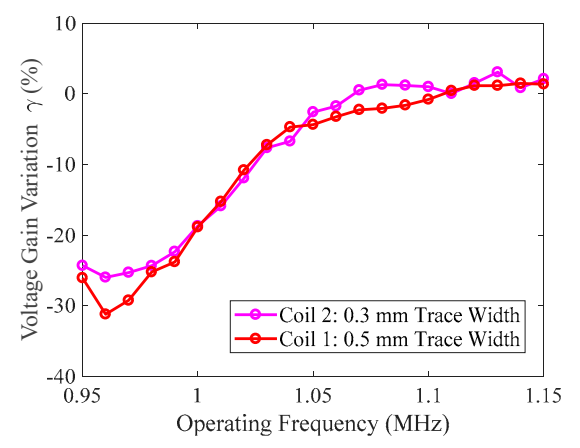

Figure 17. Measurements of $\gamma$ varying with $f$ for Coils 1,3 , and 5. 
To investigate the impact of turn numbers, Coils 1, 4, and 6 are compared. For Coil 1, $C_{\mathrm{S}}=1.97 \mathrm{nF}$ and $C_{P}=8.48 \mathrm{nF}$. For Coil $4, C_{S}=6.35 \mathrm{nF}$ and $C_{P}=25.07 \mathrm{nF}$. For Coil $6, C_{\mathrm{S}}=0.62 \mathrm{nF}$ and $C_{\mathrm{P}}=2.48 \mathrm{nF}$. Thus, $\lambda$ is around 0.2 for these three coils. The measurements of these three coils when the coin is placed at the center position are shown in Figure 18. The larger the turn number, the larger the voltage gain variation. This is because a large turn number in this case leads to a large quality factor, which helps improve the detection accuracy. Therefore, Coil 6 is selected to test the system performance.

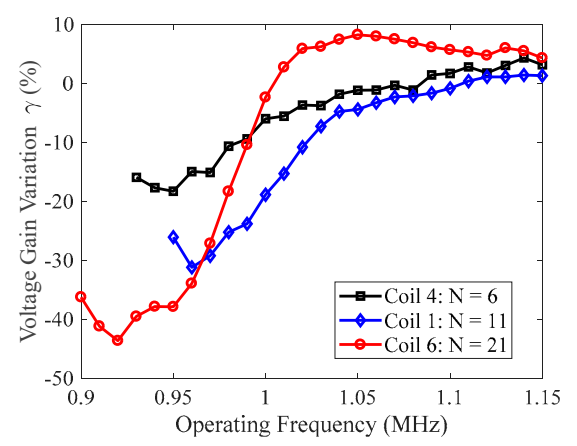

Figure 18. Measurements of $\gamma$ varying with $f$ for Coils 1,4 , and 6 .

For Coil 6, the MO is placed at two positions and two heights: namely at the center and boundary positions, and when the MO is directly attached to the detection coil $(0 \mathrm{~mm})$ and when the $\mathrm{MO}$ is $5 \mathrm{~mm}$ above the detection coil. $C_{\mathrm{S}}=0.62 \mathrm{nF}$ and $C_{\mathrm{P}}=2.48 \mathrm{nF}$. Another case is studied when there is one coin at the center position and one at the boundary. The measurements of these five cases are shown in Figure 19. We can see that even at the boundary position at $5 \mathrm{~mm}$, the voltage gain variation is about $10 \%$, significant enough to be detected. Thus, the proposed solution can cover the whole area at $5 \mathrm{~mm}$ above the detection coil. For the case with two coins, the voltage gain variation is larger than the case with one coin, as expected.

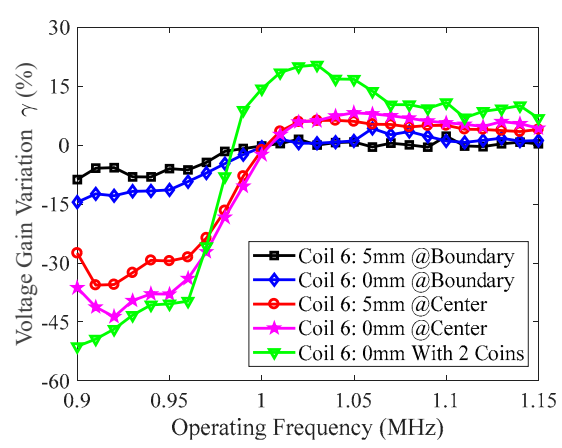

Figure 19. Measurements of $\gamma$ varying with operating frequency under different positions for Coil 6.

\subsection{System Performance}

In this Subsection, the MOD system uses the four-layer rectangle layout detection coil array and the PS mixed resonant circuit. A wireless EV charging system is implemented, and the prototype of the MOD system is shown in Figure 20. The experimental waveforms and the snapshot from the power analyzer are displayed in Figure 21.

A portion of the four-layer rectangle layout detection coil array is processed with $1 \mathrm{~mm}$ thick four-layer PCB, as shown in Figure 22. The measured inductance of a bipolar detection coil $L_{\mathrm{det}}$ is $22.18 \mu \mathrm{H}$. The series capacitance $C_{S}$ in the PS mixed resonant circuit is $0.2 \mathrm{nF}$, and the parallel capacitance $C_{P}$ in the PS mixed resonant circuit is $0.94 \mathrm{nF}$. A one-cent coin was placed and tested at various positions of the detection coil array, with the results of four positions shown in Table 2. Position 1 is at the center of the detection coil $\mathrm{A}_{12}$. Position 2 and 3 are at the edge of $\mathrm{A}_{12}$. Position 4 is at the 
vertex of $A_{12}$. The bipolar detection coils $A_{1}, B_{1}, C_{1}$, and $D_{1}$, are turned on in a certain order. When the coin was placed at the center of the detection coil, the output voltage variation was $-48.7 \%$, which is close to the calculated result in Figure 12. Even if the coin was placed at the vertex of $A_{12}$, the output voltage variation could still reach $38.3 \%$, when the detection coil $\mathrm{D}_{1}$ is turned on.

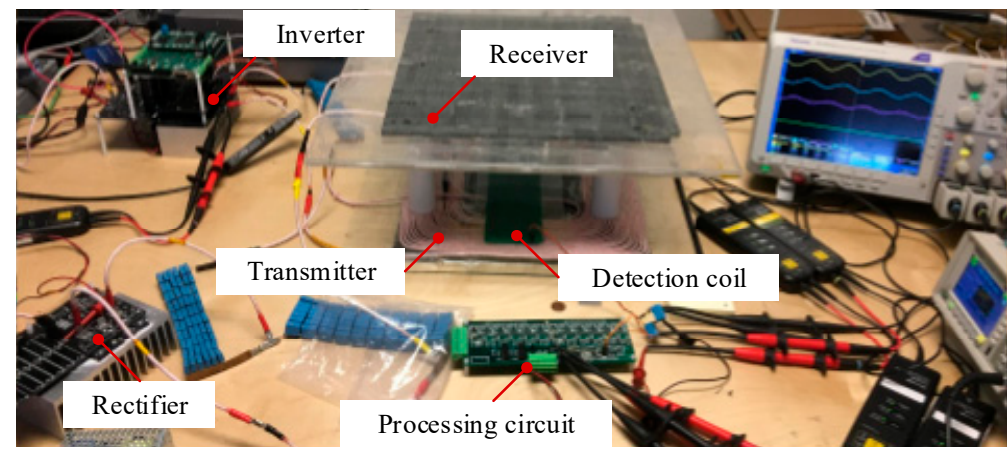

Figure 20. Photograph of wireless electric vehicle (EV) charging system with MOD system.

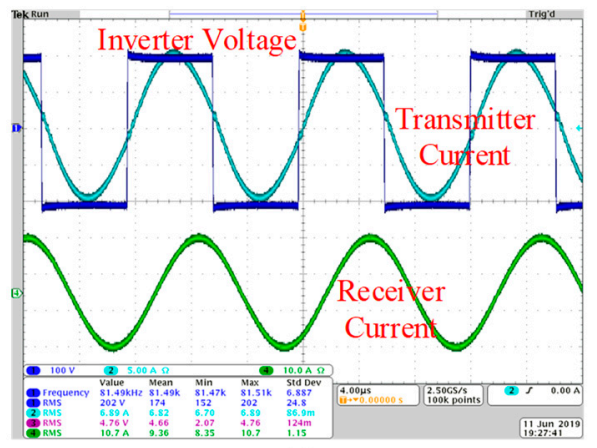

(a)

\begin{tabular}{|c|c|c|}
\hline & Without FOD & With FOD \\
\hline Udc3 & $200.29 \mathrm{~V}$ & $200.29 \mathrm{~V}$ \\
\hline Idc3 & $6.444 \quad \mathrm{~A}$ & $6.485 \mathrm{~A}$ \\
\hline $\mathrm{P} 3 \cong$ & $1.291 \mathrm{kw}$ & $1.299 \mathrm{kw}$ \\
\hline Udc1 $\overrightarrow{\underline{Q}}$ & $120.76 \mathrm{~V}$ & $120.77 \mathrm{~V}$ \\
\hline $\mid d c 1$ تु & 9.838 A & $9.887 \quad A$ \\
\hline 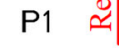 & $1.188 \mathrm{~kW}$ & $1.194 \mathrm{kw}$ \\
\hline Eff & $92.058 \%$ & $91.941 \%$ \\
\hline $\mathrm{Jdc} 2$ & $0.002 \mathrm{~V}$ & $0.002 \mathrm{~V}$ \\
\hline
\end{tabular}

(b)

Figure 21. (a) Experimental waveforms. (b) Snapshot from power analyzer.

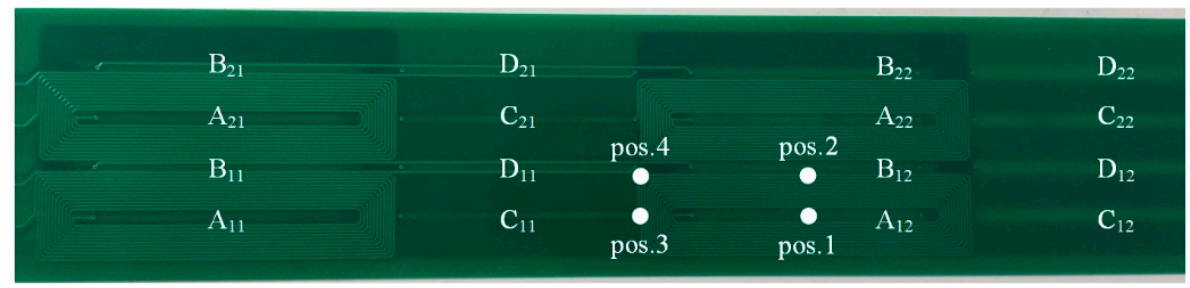

Figure 22. The PCB of the four-layer rectangle layout detection coil array. 
Table 2. $\gamma$ with the coin at different positions (pos.).

\begin{tabular}{ccccc}
\hline Coin Position & Coil $\mathbf{A}_{\mathbf{1}}$ & Coil $\mathbf{B}_{\mathbf{1}}$ & Coil $_{\mathbf{1}}$ & Coil $_{\mathbf{1}}$ \\
\hline pos. 1 & $-48.7 \%$ & $-5.1 \%$ & $/$ & $/$ \\
pos. 2 & $-8.9 \%$ & $-39.2 \%$ & $/$ & $/$ \\
pos. 3 & $-8.9 \%$ & $-2.0 \%$ & $-39.9 \%$ & $-3.0 \%$ \\
pos. 4 & $-4.8 \%$ & $-9.9 \%$ & $-5.2 \%$ & $-38.3 \%$ \\
\hline
\end{tabular}

It is difficult to accurately place MOs at a given position during the power transfer. Therefore, the MOs are firstly placed at the given position and are then removed. A quarter-dollar coin $(75 \%$ copper and $25 \%$ nickel, $24.26 \mathrm{~mm}$ in diameter, and $1.75 \mathrm{~mm}$ in thickness), a five-cent coin (75\% copper and $25 \%$ nickel, $21.21 \mathrm{~mm}$ in diameter, and $1.95 \mathrm{~mm}$ in thickness), and a one-cent coin are placed at the center of a detection coil, and then the three coins are sequentially removed from the detection coil PCB. The waveforms of the detection circuits are as shown in Figure 23. As the coins are removed one by one, the amplitudes of $U_{\operatorname{det}}$ and $U_{\text {out }}$ also increase significantly one by one. The inductance variations of the detection coils caused by three coins, two coins, and one coin are different, so the test result indicates the MOD system can detect various inductance variations.

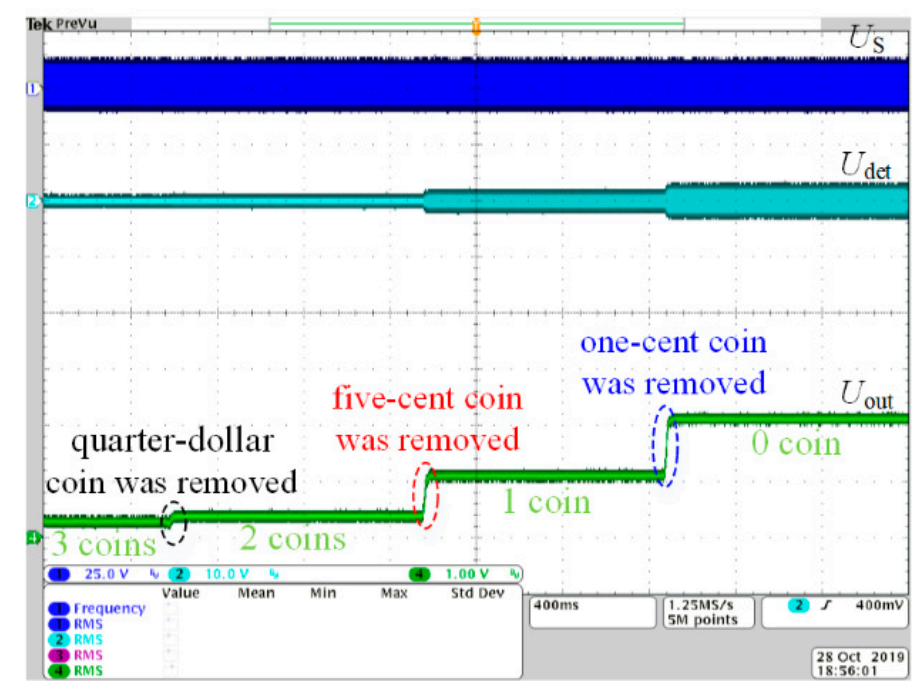

Figure 23. Waveforms of detection circuits when three coins were sequentially removed.

\section{Conclusions}

In this paper, the MOD systems suitable for wireless EV charging were proposed based on inductive sensing in the field-based FOD method.

Compared with the single-layer detection coils, the novel three-layer hexagonal layout and four-layer rectangle layout detection coil structures can cover the whole area of the TX pad without blind spots, which can further improve the detection performance of the MOD system. Two adjacent unipolar coils connected in series form a bipolar coil so as to decouple the detection coils from the TX. Compared with the three-layer hexagonal layout detection coils, the four-layer rectangular layout detection coils have a slightly larger potential for eliminating blind spots. But, for lower cost and easier routing and wiring, the three-layer hexagonal layout detection coil structure will be a better choice.

The SP and PS mixed resonant circuits were proposed in this paper to amplify the inductance variation for better detection performance. These two mixed resonances have the larger impedance amplitude variation than the parallel resonant circuit and maintain the advantage of large impedance amplitude at the same time. Furthermore, the methods to select the proper combination of series and parallel capacitors and the methods to select the proper operating frequency are presented in this paper to enhance the resonant circuits further. 
The impacts of the detection coil layer, trace width, and turn-number are investigated based on the three-layer hexagonal layout detection coils. The upper layer, the larger trace width, and a larger turn-number all have larger voltage variation. The test of the MOD system shows that the one-cent coin can be detected at various positions both on the three-layer and four-layer detection coil PCB. Even if the coin was placed at the worst position of the detection coils, the output voltage variation could still reach $38.3 \%$, and the MOD system can detect various inductance variations without blind spots.

Author Contributions: B.C. and Y.Z. are the main authors of this manuscript; R.C., J.L. and G.P. made suggestions for this work; C.C.M. reviewed and edited the draft. All authors have read and agreed to the published version of the manuscript.

Funding: This research was funded by a grant from Hyundai Motor Company.

Conflicts of Interest: The authors declare no conflict of interest.

\section{References}

1. Hui, S.R. Technical and safety challenges in emerging trends of near-field wireless power transfer industrial guidelines. IEEE Electromagn. Compat. Mag. 2018, 7, 78-86. [CrossRef]

2. Bi, Z.; Kan, T.; Mi, C.C.; Zhang, Y.; Zhao, Z.; Keoleian, G.A. A review of wireless power transfer for electric vehicles: Prospects to enhance sustainable mobility. Appl. Energy 2016, 179, 413-425. [CrossRef]

3. Covic, G.A.; Boys, J.T. Inductive Power Transfer. Proc. IEEE 2013, 101, 1276-1289. [CrossRef]

4. Kan, T.; Zhang, Y.; Yan, Z.; Mercier, P.P.; Mi, C. A Rotation-Resilient Wireless Charging System for Lightweight Autonomous Underwater Vehicles. IEEE Trans. Veh. Technol. 2018, 67, 6935-6942. [CrossRef]

5. Yuan, L.; Li, B.; Zhang, Y.; He, F.; Chen, K.; Zhao, Z. Maximum efficiency point tracking of the wireless power transfer system for the battery charging in electric vehicles. In Proceedings of the 2015 18th International Conference on Electrical Machines and Systems (ICEMS), Institute of Electrical and Electronics Engineers (IEEE), Pattaya, Thailand, 25-28 October 2015; pp. 1101-1107.

6. Liu, C.; Jiang, C.; Song, J.; Chau, K.T. An Effective Sandwiched Wireless Power Transfer System for Charging Implantable Cardiac Pacemaker. IEEE Trans. Ind. Electron. 2018, 66, 4108-4117. [CrossRef]

7. Huang, Z.; Wong, S.C.; Tse, C.K. Control Design for Optimizing Efficiency in Inductive Power Transfer Systems. IEEE Trans. Power Electron. 2018, 33, 4523-4534. [CrossRef]

8. Patil, D.; McDonough, M.K.; Miller, J.M.; Fahimi, B.; Balsara, P.T. Wireless Power Transfer for Vehicular Applications: Overview and Challenges. IEEE Trans. Transp. Electr. 2018, 4, 3-37. [CrossRef]

9. Onar, O.C.; Campbell, S.; Seiber, L.E.; White, C.P.; Chinthavali, M. A high-power wireless charging system development and integration for a Toyota RAV4 electric vehicle. In Proceedings of the 2016 IEEE Transportation Electrification Conference and Expo (ITEC), Institute of Electrical and Electronics Engineers (IEEE), Dearborn, MI, USA, 27-29 June 2016; pp. 1-8.

10. Guo, Y.; Wang, L.; Zhang, Y.; Li, S.; Liao, C. Rectifier Load Analysis for Electric Vehicle Wireless Charging System. IEEE Trans. Ind. Electron. 2018, 65, 6970-6982. [CrossRef]

11. Widmer, H.; Sieber, L.; Daetwyler, A.; Bittner, M. Systems, Methods, and Apparatus for Radar-Based Detection of Objects in a Predetermined Space. U.S. Patent 9,772,401 B2, 26 September 2017.

12. Sieber, L.; Chopra, S. Systems, Methods, and Apparatus for Living Object Protection in Wireless Power Transfer Applications. U.S. Patent 2016/0109564 A1, 21 April 2016.

13. Poguntke, T.; Schumann, P.; Ochs, K. Radar-based living object protection for inductive charging of electric vehicles using two-dimensional signal processing. Wirel. Power Transf. 2017, 4, 88-97. [CrossRef]

14. Bell, D.; Leabman, M.A. Systems and Methods of Object Detection Using One or More Sensors in Wireless Power Charging Systems. U.S. Patent 10,483,768, 16 January 2018.

15. Hoffman, P.F.; Boyer, R.J.; Henderson, R.A. Foreign Object Detection System and Method Suitable for Source Resonator of Wireless Energy Transfer System. U.S. Patent 9,304,042 B2, 5 April 2016.

16. Karanth, A.; Dorairaj, H.H.K.; Balakrishnan, R.; Kumar, R. Foreign Object Detection in Inductive Coupled Wireless Power Transfer Environment Using Thermal Sensors. U.S. Patent 2013/0163635 A1, 27 June 2013. 
17. Fukuda, S.; Nakano, H.; Murayama, Y.; Murakami, T.; Kozakai, O.; Fujimaki, K. A novel metal detector using the quality factor of the secondary coil for wireless power transfer systems. In Proceedings of the 2012 IEEE MTT-S International Microwave Workshop Series on Innovative Wireless Power Transmission: Technologies, Systems, and Applications, Tokyo, Japan, 10-11 May 2012; pp. 241-244. [CrossRef]

18. Jeong, S.Y.; Kwak, H.G.; Jang, G.C.; Choi, S.Y.; Rim, C.T.; Kwak, H.G. Dual-Purpose Nonoverlapping Coil Sets as Metal Object and Vehicle Position Detections for Wireless Stationary EV Chargers. IEEE Trans. Power Electron. 2017, 33, 7387-7397. [CrossRef]

19. Zhang, H.; Ma, D.; Lai, X.; Yang, X.; Tang, H. The Optimization of Auxiliary Detection Coil for Metal Object Detection in Wireless Power Transfer. In Proceedings of the 2018 IEEE PELS Workshop on Emerging Technologies: Wireless Power Transfer (Wow), Montréal, QC, Canada, 3-7 June 2018; pp. 1-6. [CrossRef]

20. Xiang, L.; Zhu, Z.; Tian, J.; Tian, Y. Foreign Object Detection in a Wireless Power Transfer System Using Symmetrical Coil Sets. IEEE Access 2019, 7, 44622-44631. [CrossRef]

21. Sieber, L.; Fischer, M.; Widmer, H. Systems, Methods, and Apparatus for Increased Foreign Object Detection Loop Array Sensitivity. U.S. Patent 9,921,045 B2, 20 March 2018.

22. Jeong, S.Y.; Kwak, H.G.; Jang, G.C.; Rim, C.T. Living object detection system based on comb pattern capacitive sensor for wireless EV chargers. In Proceedings of the 2016 IEEE 2nd Annual Southern Power Electronics Conference (SPEC), Institute of Electrical and Electronics Engineers (IEEE), Auckland, New Zealand, 5-8 December 2017; pp. 1-6.

23. Jeong, S.Y.; Thai, V.X.; Park, J.H.; Rim, C.T. Self-Inductance-Based Metal Object Detection With Mistuned Resonant Circuits and Nullifying Induced Voltage for Wireless EV Chargers. IEEE Trans. Power Electron. 2018, 34, 748-758. [CrossRef]

24. Chopra, S. Percebon, L. Dynamic Mutual Sensing Foreign Object Detection Loops. U.S. Patent 2018/0076671 A1, 15 March 2018.

25. Khan, S.R.; Pavuluri, S.K.; Desmulliez, M.P. Accurate Modeling of Coil Inductance for Near-Field Wireless Power Transfer. IEEE Trans. Microw. Theory Tech. 2018, 66, 4158-4169. [CrossRef]

(C) 2020 by the authors. Licensee MDPI, Basel, Switzerland. This article is an open access article distributed under the terms and conditions of the Creative Commons Attribution (CC BY) license (http://creativecommons.org/licenses/by/4.0/). 